\title{
Patient with Acute Shortness of Breath, Elevated D-Dimer, and a Previous History of Cancer*
}

Katie Traylor and Paresh Mahajan

Department of Diagnostic Radiology and Molecular Imaging, Oakland University William Beaumont School of Medicine, Royal Oak, Michigan

\begin{abstract}
A 69-y-old man with a history of small cell lung cancer, esophageal cancer, and pulmonary hypertension stated that he had shortness of breath. The D-dimer level was elevated at 1,385 .

The patient inhaled a calculated $51.8 \mathrm{MBq}(1.4 \mathrm{mCi})$ of ${ }^{99 \mathrm{~m}} \mathrm{Tc}$-diethylenetriminepentaacetic acid aerosol, after which planar images of the lungs in the standard 8 projections were obtained. After intravenous administration of $151.7 \mathrm{MBq}(4.1$ $\mathrm{mCi}$ ) of ${ }^{99 \mathrm{~m}} \mathrm{Tc}-$ labeled macroaggregated albumin (MAA), a set of projections corresponding to those obtained on the ventilation study was obtained (Fig. 1).
\end{abstract}

FIGURE 1. (Top) Perfusion images of lungs. Views from left to right are anterior/posterior, lateral, right anterior oblique/left posterior oblique, and left anterior oblique/right posterior oblique. (Bottom) Ventilation images in projections corresponding to perfusion images.

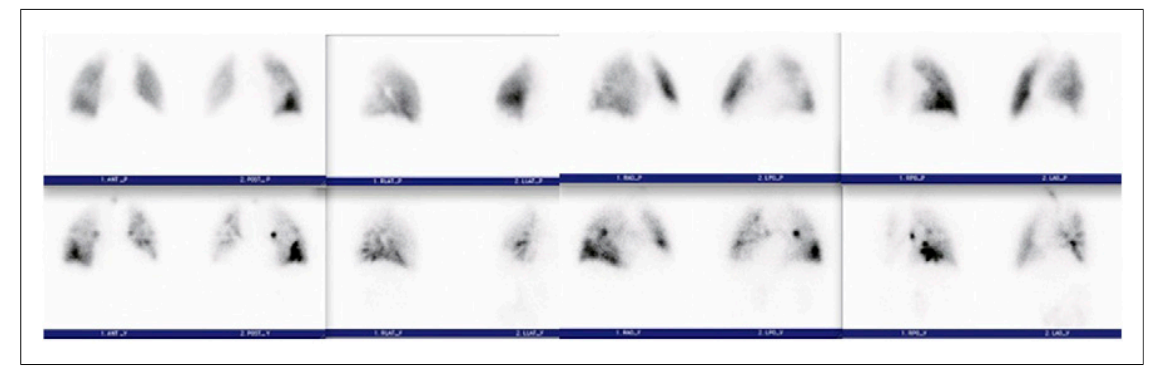

\section{QUESTION 1}

This patient has a history of pulmonary hypertension. What difference in the technique had to be addressed before the study was performed? Why?

A. Reduction of the number of ${ }^{99 m}$ Tc-MAA particles to less than 100,000 to lessen the likelihood of blockade of the capillaries.

B. Reduction of the ${ }^{99 \mathrm{~m}} \mathrm{Tc}$ dose to decrease the radiation dose to the patient.

C. Completion of perfusion images first. If these are normal, the ventilation images do not need to be completed, thus decreasing the radiation dose to the patient.

D. Reduction of the number of ${ }^{99 \mathrm{~m} T c-M A A}$ particles to $100,000-200,000$ to lessen the likelihood of blockade of the capillaries.

FIGURE 2. Chest posteroanterior radiograph that was completed approximately $12 \mathrm{~h}$ before lung scan.

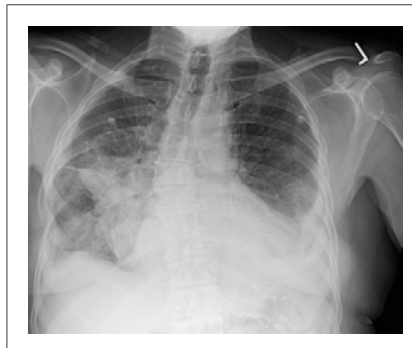

\section{QUESTION 2}

After reviewing the lung scan images and comparing them to the chest radiograph (Fig. 2), what is your assessment of the findings based on the modified PIOPED II interpretation criteria (Table 1)?

A. High probability of pulmonary embolism, as there are 2 large segmental mismatched defects in the right lower lobe.

B. Nondiagnostic scan for pulmonary embolism, as there is 1 moderate segmental triple match in the superior segment of the right lower lobe.

Published online Jul. 18, 2013.

DOI: $10.2967 /$ jnmt.113.126755

COPYRIGHT @ 2013 by the Society of Nuclear Medicine and Molecular Imaging, Inc. 
TABLE 1

Ventilation, Perfusion, and Radiographic Interpretive Criteria for Pulmonary Embolism

\begin{tabular}{|c|c|c|c|}
\hline PIOPED & Modified PIOPED II & $\begin{array}{c}\text { Perfusion-only modified } \\
\text { PIOPED II }\end{array}$ & $\begin{array}{l}\text { Perfusion-only } \\
\text { PISAPED }\end{array}$ \\
\hline High LR & High LR & PE present & PE present \\
\hline $\begin{array}{l}>2 \text { large mismatched }(\mathrm{V}: \mathrm{Q}) \\
\text { segmental defects* }\end{array}$ & $\begin{array}{l}\geq 2 \text { large mismatched }(\mathrm{V}: \mathrm{Q}) \\
\text { segmental defects }^{\star}\end{array}$ & $\begin{array}{l}\geq 2 \text { large mismatched } \\
\text { (Q:CXR) segmental } \\
\text { defects }^{\star}\end{array}$ & $\begin{array}{l}\geq 1 \text { wedge-shaped } \\
Q \text { defects }\end{array}$ \\
\hline \multicolumn{4}{|l|}{ Borderline high LR } \\
\hline \multicolumn{4}{|l|}{$\begin{array}{l}2 \text { large mismatched }(\mathrm{V}: \mathrm{Q}) \\
\text { segmental defects }\end{array}$} \\
\hline Intermediate LR & Nondiagnostic & Nondiagnostic & Nondiagnostic \\
\hline $\begin{array}{l}2 \text { moderate or } 1 \text { large mismatched } \\
(\mathrm{V}: \mathrm{Q}) \text { defect } \\
\text { Difficult to categorize as high or low }\end{array}$ & All other findings & All other findings & $\begin{array}{l}\text { Cannot classify as } \\
\text { PE-present or } \\
\text { PE-absent }\end{array}$ \\
\hline \multicolumn{4}{|l|}{ Borderline low LR } \\
\hline \multicolumn{4}{|l|}{1 matched (V:Q) defect, CXR-negative } \\
\hline \multicolumn{4}{|l|}{ Low LR } \\
\hline \multicolumn{4}{|l|}{$\begin{array}{l}\text { Nonsegmental perfusion defects } \\
Q \text { defect substantially }<\text { CXR defect } \\
\text { Matched (V:Q) defects, CXR-negative } \\
\text { Any number of small } Q \text { defects }{ }^{*}\end{array}$} \\
\hline Normal & Very low LR & PE absent & PE absent \\
\hline \multirow[t]{3}{*}{ No $Q$ defects } & $\begin{array}{l}\text { Nonsegmental }{ }^{\dagger} \\
\mathrm{Q} \text { defect }<\mathrm{CXR} \text { lesion } \\
\text { 1-3 small segmental } \\
\text { defects } \\
\text { Solitary matched }(\mathrm{V}: \mathrm{Q}: \mathrm{CXR}) \\
\quad \text { defect ( } 1 \text { segment) in mid } \\
\text { or upper lung } \\
\text { Stripe sign } \\
\text { Solitary large pleural } \\
\text { effusion } \$ \\
\geq 2 \text { matched }(\mathrm{V}: \mathrm{Q}) \text { defects, } \\
\text { regionally normal CXR }\end{array}$ & $\begin{array}{l}\text { Very low probability } \\
\text { Nonsegmental } \\
\text { Q defect }<\mathrm{CXR}^{\dagger} \text { lesion } \\
\text { 1-3 small segmental } \\
\quad \text { defects } \\
\text { Solitary matched (Q:CXR) } \\
\text { defect ( } \leq 1 \text { segment) in } \\
\text { mid or upper lung } \\
\text { Stripe sign } \\
\text { Solitary large pleural } \\
\text { effusion } \$\end{array}$ & $\begin{array}{l}\text { Non-wedge-shaped } \\
\text { Q defect } \\
\text { Contour defect caused } \\
\text { by enlarged heart, } \\
\text { mediastinum, or } \\
\text { diaphragm } \\
\text { Near-normal Q } \\
\text { Normal Q }\end{array}$ \\
\hline & Normal & & \\
\hline & No $Q$ defects & & \\
\hline
\end{tabular}

\footnotetext{
${ }^{*}$ Or equivalent where large segmental defect, $>75 \%$ of segment, equals 1 segmental equivalent; moderate defect, $25 \%-75 \%$ of segment, equals 0.5 segmental equivalent; small defect, $<25 \%$, is not counted.

${ }^{\dagger}$ For example, prominent hilum, cardiomegaly, elevated diaphragm, linear atelectasis, or costophrenic angle effusion with no other perfusion defect in either lung and no other radiographic lesion.

${ }^{\ddagger}$ Peripheral perfusion in a defect (best seen on tangential view).

§Pleural effusion in at least one third of pleural cavity, with no other perfusion defect in either lung.

"Perfusion defects exactly match shape of CXR.

$\mathrm{V}: \mathrm{Q}$ = ventilation-perfusion; $\mathrm{CXR}=$ chest radiograph; $\mathrm{PE}=$ pulmonary embolism; $\mathrm{LR}=$ likelihood ratio.
}

C. Low probability of pulmonary embolism.

D. Nondiagnostic scan for pulmonary embolism, as there is 1 large segmental triple match in the superior segment of the right lower lobe, although more information would be valuable for an accurate interpretation.

\section{QUESTION 3}

The patient also underwent CT of the chest with intravenous contrast material (Fig. 3) within the last week. Do the CT findings change your previous diagnosis?

A. No, the patient still has a high probability of pulmonary embolism.

B. No, the patient still has a nondiagnostic scan for pulmonary embolism.

C. The scan is technically nondiagnostic; however, it appears that the patient has postsurgical changes within the opaque region identified on the chest radiograph.

D. No, the patient still has a high probability for pulmonary embolism even though there are postsurgical changes within the opaque region identified on the chest radiograph. 


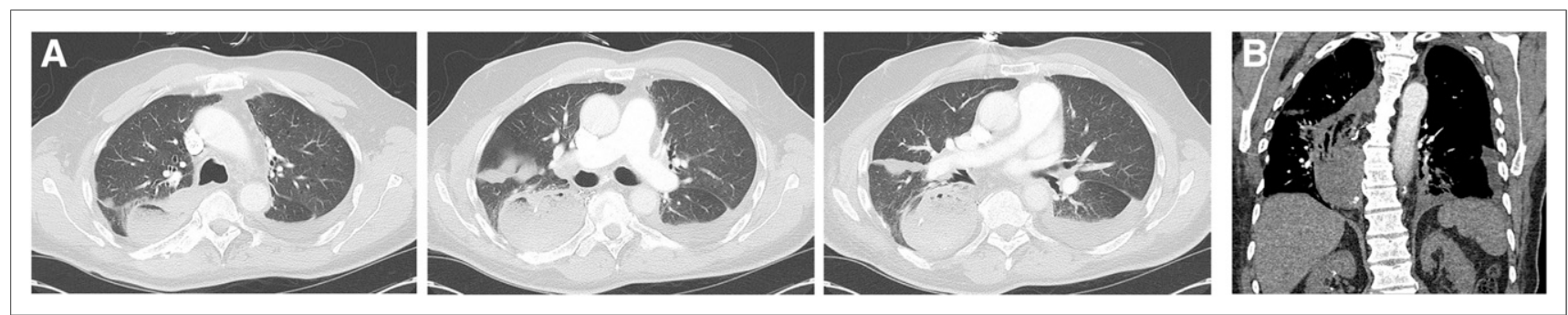

FIGURE 3. Contrast-enhanced chest CT scan. (A) From left to right: 3 axial slices (lung window) from cranial to caudal. (B) Coronal slice (soft-tissue window) illustrating extent of gastric pull-through, which caused large defect on ventilation-perfusion images.

\section{QUESTION 4}

Besides lymph node involvement and distant metastatic disease, what additional information is needed for staging purposes?

A. The lymph node location is needed because lymph nodes above and below the diaphragm may change the stage of the disease.

B. The endoscopic ultrasonography and pathology report is needed because the extent of invasion of the cancer can change the stage of the disease.

C. Whether a lymph node is present in the supraclavicular regions needs to be known because the patient would be deemed a nonsurgical candidate.

D. The location of the distant metastatic disease is needed because this information may change the stage of the patient.

\section{DISCUSSION}

Knowing the entire history of a patient is important when reviewing studies, especially when the patient may have postsurgical changes that may interfere with one's interpretation. Patients with benign and malignant disease of their esophagus may undergo radical resection and reconstruction with an intrathoracic stomach, colon, or jejunum pulled up through the mediastinum, retrosternally or subcutaneously. These postsurgical changes will appear as a widened mediastinum, usually on the right side. The history is important, as air-space disease, mass lesions, pleural effusion, or lobar collapse may look the same as one another on a chest radiograph. Knowing the history of our patient, we were able to relay this information to the referring physician and communicate that the mismatch was most likely due to the patient's gastric pullthrough and radiotracer activity located within the stomach. Strictly speaking, with the PIOPED criteria the patient would still remain at intermediate probability of pulmonary embolism because the corresponding ventilation-perfusion defects correlated with the parenchymal opacity seen in the lower lung zone on the chest radiograph. The PIOPED criteria look only at comparing the lung scan to the chest radiograph and not any other helpful imaging, such as the CT scan of the chest.

\section{BIBLIOGRAPHY}

Brunicardi FC, Anderson DK, Billiar T, et al. Schwartz's Principles of Surgery. 8th ed. New York, NY: McGraw-Hill Professional; 2005 :888-893.

Cameron JL. Current Surgical Therapy. 8th ed. Maryland Heights, MO: Mosby; 2004:44-49.

Cole TJ, Turner MA. Manifestations of gastrointestinal disease on chest radiographs. Radiographics. 1993;13:1013-1034.

Krasna MJ. Multimodality therapy for esophageal cancer. Oncology. 2010;24:1134-1138.

Mettler FA, Guiberteau MJ. Essentials of Nuclear Medicine Imaging. 6th ed. Philadelphia, PA: Elsevier Saunders; 2012:197, 213-216, 223.

Society of Nuclear Medicine Procedure Guideline for Lung Scintigraphy. SNMMI Web site. http://interactive.snm.org/docs/Lung_Scintigraphy_V4_Final.pdf. Published March 2012. Accessed June 24, 2013.

*For the answers, see page 242. 


\section{Question 1}

\section{Answers to the Questions on Pages 234 and 235}

Answer: C

A horizontal orientation of the sacrum is one of the major findings in patients with achondroplasia. On the posterior planar images, this orientation causes the sacrococcygeal region to point more posteriorly than in subjects without achondroplasia, creating the appearance of increased radiotracer uptake. The addition of SPECT or SPECT/ CT helps differentiate physiologic variations from a true abnormality.

A. The patient reported no recent trauma, so a fracture is unlikely.

B. The patient did not have any risk factors for osteomyelitis in the sacrococcygeal region, such as sacral decubital ulcers. If osteomyelitis was suspected, a triple-tracer study (bone scan, sulfur colloid, and indium-labeled white blood cells) could be performed.

D. Osteoid osteomas do present with focal intensely increased radiotracer uptake on bone scans. Patients typically complain of bone pain that is worse at night, and blood-flow and blood-pool images often demonstrate intense activity.

\section{Question 2}

\section{Answer: A}

Achondroplasia can indeed be diagnosed on prenatal ultrasound. Sonography will often demonstrate a rounded metaphyseal-epiphyseal interface, with an angle connection to diaphysis wider than expected, sometimes referred to as the collar-hoop sign.

B. Achondroplasia is the most common cause of dwarfism.

C. It is most often diagnosed on prenatal ultrasound or on the basis of clinical and radiographic findings.

D. The extremities are disproportionately shortened in comparison to the axial skeleton, which often is actually normal in size.

\section{Question 3}

Answer: B

There are typically no intellectual deficits associated with achondroplasia. Most people with achondroplasia have a normal IQ.

A. Narrowing of the spinal canal is the pathologic hallmark.

C. The champagne glass pelvis, which is due to anteversion of the pelvis with squared-off ilia and a low anterior superior iliac spine and high posterior superior iliac spine, is another radiographic feature of achondroplasia.

D. A depressed nasal bridge and a prominent forehead are two of the major facial features.

\section{Question 4}

\section{Answer: D}

Because foramen magnum stenosis can lead to significant complications, including apnea, cervicomedullary decompression is often necessary.

A. Genu varus, not valgus, is seen in achondroplasia.

B. Pulmonary hypertension is not a known association.

C. Infertility is not a feature of achondroplasia.

\section{Question 1}

\section{Answers to the Questions on Pages 236 and 237}

Answer: D

A. Tracer is trapped in the left lung during the washout phase of the ${ }^{133} \mathrm{Xe}$ ventilation images; the findings can be due to either severe chronic obstructive pulmonary disease or bronchopleural fistula.

B. The findings can be due to either chronic obstructive pulmonary disease or bronchopleural fistula.

C. The scan findings are abnormal because of trapping of the tracer during the washout phase of the ${ }^{133} \mathrm{Xe}$ ventilation scan.

D. The findings can be due to either chronic obstructive pulmonary disease or bronchopleural fistula. 


\section{Question 2}

Answer: C

${ }^{133} \mathrm{Xe}$ is a radioactive gas and rapidly clears from the body mostly by exhalation, with a biologic half-life of $30 \mathrm{~s}$.

\section{Question 3}

\section{Answer: D}

CT is a helpful modality in visualizing a bronchopleural fistula. CT identifies the location, number, size, and etiology of the fistula. Communication between the airway and pleural space can be seen on thin-section CT. Other associated findings on CT can be lung consolidation, pneumothorax, and empyema or an air-fluid level in the pleural space.

\section{Question 1}

\section{Answers to the Questions on Pages 238-240}

\section{Answer: D}

${ }^{99 m}$ Tc-MAA is the radiopharmaceutical used in pulmonary perfusion imaging. The ${ }^{99 m} \mathrm{Tc}-$ MAA particles localize by capillary embolization and blockade. Generally, of the total network of capillaries in the lung, only a miniscule amount are actually embolized. Therefore, this is rarely of clinical concern. Without the presence of shunts, $95 \%$ of the particles are removed from the circulation on the first pass through the pulmonary capillary beds. The particle size will usually range from 5 to $100 \mu \mathrm{m}$, with most being in the range of 10-30 $\mu \mathrm{m}$. Severe pulmonary hypertension is a relative contraindication to performing particulate perfusion lung scanning because of the blockade of additional capillaries. This may acutely exacerbate the patient's condition and the patient's cardiac complications. It is prudent to use a freshly prepared ${ }^{99 \mathrm{~m}} \mathrm{Tc}-\mathrm{MAA}$ kit and reduce the number of injected particles to $100,000-200,000$. Optimally, a perfusion lung scan should have 200,000-600,000 particles. If the particles are reduced to below 100,000, inhomogeneity in the images may lead to incorrect interpretation.

$\mathrm{C}$ is not correct. This technique modification may be performed on a pregnant patient to decrease the radiation dose to the fetus. The biologic half-life for ${ }^{99} \mathrm{mTc}-\mathrm{MAA}$ in the lung is $2-4 \mathrm{~h}$. The normal administered activity to adults is $74-148 \mathrm{MBq}(2-4 \mathrm{mCi})$. The lung is the critical organ, and the absorbed dose to the lung is approximately 5 $\mathrm{mGy}(0.5 \mathrm{rad})$ for a $74-\mathrm{MBq}(3-\mathrm{mCi})$ dose.

\section{Question 2}

Answer: D

The perfusion defect is large but somewhat unusual in shape, especially in the inferior aspect, and appears mostly mismatched. On the patient's chest radiograph, surgical clips are noted along the right lower lung opacity. Therefore, it is not known, without further information, whether this finding is due to an acute pulmonary process or to postsurgical changes alone. The PIOPED criteria portion of this question will be addressed in the answer of the next question.

\section{Question 3}

Answer: C

This patient has a history of a gastric pull-through due to his esophageal cancer (Fig. 3B). From the CT scan of the chest, it is evident that the stomach has been pulled into the right hemithorax, accounting for the defect noted in this region on the perfusion and ventilation images. The large area of diffusely reduced uptake at the posteromedial right lung is best depicted on the posterior images and appears lunar in configuration, corresponding to the findings on the coronal image of the chest CT scan (Fig. 3B). On the right posterior oblique image (Fig. 1), the defect appears to be somewhat wedge-shaped and is localized approximately to the posterior segment of the superior segment of the right lower lobe. The ventilation portion of this study shows a mild reduction in this region with focally increased uptake, although this uptake reflects swallowed radiotracer activity within the stomach, a common finding. Additionally, significant clumping of the radiotracer is seen, and increased uptake projecting at the posterior basal segment of the right lower lobe on the posterior image of the ventilation study "shines through" on the subsequent perfusion portion of the scan. There is also central clumping of radiotracer, which could reflect a component of obstructive airway disease. When the modified PIOPED II criteria are taken into account, the patient can be said to have a nondiagnostic scan for pulmonary embolism since the corresponding ventilation-perfusion defect is located at the opacity seen in the lower lung fields on the chest radiograph. This study was read as having a very low likelihood ratio since the defect corresponds to the nonpulmonary defect of the gastric pull-through. It was recommended that if pulmonary 
embolism remained a clinical concern, anticoagulation with heparin might be initiated and the ventilation images could be repeated—only, however, after the patient had ingested a significant amount of water after inhaling the aerosol to help wash the radiotracer from the intrathoracic stomach. Alternatively, CT pulmonary angiography may be performed.

\section{Question 4}

\section{Answer: B}

The staging system for esophageal cancer is based on the TNM system of the American Joint Committee on Cancer, with increases in the T stage being based on the degree of invasion of the esophageal cancer. In this new staging system, $\mathrm{N}$ stage is determined by number of lymph nodes rather than by location of the lymph node, as was done in the previous staging criteria.

A. Lymph node involvement above the diaphragm versus below the diaphragm is important in lymphoma staging.

B. The presence of a lymph node in the supraclavicular region does not change the stage in esophageal cancer. If a patient has no regional lymph node metastasis, the patient is considered NO, whereas if a patient has regional lymph node metastasis, the patient is considered N1. Supraclavicular lymph node involvement is important in lung cancer, and involvement of either the ipsilateral or the contralateral supraclavicular nodes would deem the patient a nonsurgical candidate.

C. In esophageal cancer staging, if the patient is M1, M1a, or M1b the patient is still considered to be stage IV. M0 indicates no distant metastasis; M1, distant metastasis; M1a, invasion of celiac or cervical lymph nodes; and M1b, other distant metastasis. 